\title{
Considerações Sobre a Habilidade de Compreensão em Leitura e Formas de sua Avaliação
}

\author{
Katya Luciane de Oliveira \\ Universidade Estadual de Londrina - Londrina - PR - Brasil \\ Patrícia Silva Lúcio \\ Universidade Estadual de Londrina - Londrina - PR - Brasil \\ Fabiano Koich Miguel \\ Universidade Estadual de Londrina - Londrina - PR - Brasil
}

\begin{abstract}
Resumo
A compreensão leitora é uma atividade complexa que envolve mecanismos cognitivos, linguísticos, sociais e emocionais. Nas sociedades ocidentais, o êxito escolar muito se relaciona a uma apreensão adequada do processo de ler e compreender o texto lido, sendo um dos requisitos para a aprendizagem. Assim, a compreensão da leitura é assunto relevante àqueles que trabalham com a educação e com problemas dela decorrentes. Este trabalho se insere nesse contexto e objetiva fornecer uma exposição geral sobre o estudo da compreensão leitora, particularmente em seus aspectos históricos, conceituais e clínicos. Primeiramente, faz-se um breve relato histórico da leitura no Brasil e de algumas questões centrais atuais. Em seguida, descreve-se a compreensão leitora e seu processamento na abordagem da psicologia cognitiva. Em terceiro, são resenhados os principais instrumentos internacionais e brasileiros de avaliação da compreensão em leitura. Finalmente, discutese a urgência de instrumentos psicometricamente ajustados para o campo da avaliação psicoeducacional.
\end{abstract}

Palavras-chave: leitura; compreensão da leitura; avaliação.

\section{Considerations about Understanding ability in reading and Ways of its Assessment}

\begin{abstract}
The reading comprehension is a complex activity that involves cognitive, linguistic, social and emotional mechanisms. In Western societies, academic achievement much relates to an adequate grasp of the process to read and understand the text read, one of the requirements for learning. Therefore, reading comprehension is relevant subject to those that work with education and with problems arising from it. This work is part of this context and aims to provide a general presentation about the study of reading comprehension, particularly in its historical, conceptual and clinical aspects. First, it is a brief reading of the historical record in Brazil and some current core issues. Then describes the reading comprehension and processing in cognitive psychology approach. Third, the main international instruments and Brazilian assessment of reading comprehension are also reviewed. Finally, we discuss the urgency of psychometrically adjusted instruments to the field of psychoeducational assessment.
\end{abstract}

Keywords: reading; reading comprehension; evaluation.

\section{Consideraciones Sobre la Habilidad de Comprensión en Lectura y Formas de su Evaluación}

\begin{abstract}
Resumen
La comprensión lectora es una actividad complexa que involucran mecanismos cognitivos, lingüísticos, sociales y emocionales. En las sociedades occidentales, el éxito escolar mucho se relaciona a una aprensión adecuada del proceso de leer y comprender el texto leído, siendo uno de los requisitos para el aprendizaje. Así, la comprensión de la lectura es tema relevante a aquellos que trabajan con la educación y con problemas derivados de ella. Este estudio se inserta en ese contexto y objetiva fornecer una exposición general sobre el estudio de la comprensión lectora, particularmente en sus aspectos históricos, conceptuales y clínicos. Primeramente, se hace un breve relato histórico de la lectura en Brasil y de algunas cuestiones centrales actuales. Enseguida, se describe la comprensión lectora y se procesamiento en el abordaje de la psicología cognitiva. E tercero, se reseña los principales instrumentos internacionales y brasileños de evaluación de la comprensión en lectura. Finalmente, se discute la urgencia de instrumentos psicométricamente ajustados para el campo de la evaluación psicoeducativo.
\end{abstract}

Palabras clave: lectura; comprensión da lectura; evaluación. 


\section{Introdução}

Um dos grandes desafios do processo de aquisição da leitura constitui a apreensão significativa daquilo que se lê, dentro e fora do contexto acadêmico. Isto porque a compreensão da leitura é ao mesmo tempo um processo e um produto de interação complexa entre o leitor (que traz para a situação suas características linguísticas, cognitivas e de conhecimento de mundo) e o texto (que apresenta propriedades que interferem na capacidade de entendimento do conteúdo a ser lido, como a "legibilidade" - readability - e a frequência vocabular). Esta complexidade dificulta o diagnóstico dos problemas de compreensão textual, tanto por parte dos educadores, quanto de psicólogos e fonoaudiólogos.

Tendo em vista a relevância deste tema para aqueles que investigam ou atuam na área dos problemas de aprendizagem, o presente trabalho visa traçar uma exposição geral sobre o estudo da compreensão leitora e suas formas de avaliação. Primeiramente, serão sintetizados os aspectos históricos e os pontos de vista atuais sobre a leitura no contexto brasileiro. Em seguida, será feito um esforço de caracterização da compreensão leitora a partir de sua forma de processamento, dentro do paradigma da psicologia cognitiva. Posteriormente, será discutido o processo de avaliação da compreensão, revisando-se, desse modo, os principais instrumentos diagnósticos atualmente disponíveis nos contextos internacional e nacional. Finalmente, nas considerações finais, buscar-se-á integrar as informações discutidas ao longo trabalho, ressaltando-se a importância do tema dentro da psicologia psicoeducacional.

\section{Aspectos Históricos e Atuais Acerca da Leitura no Brasil}

A história mundial aponta que inicialmente a leitura era privilégio de uma elite dominante. O sacerdócio também era uma maneira de se ter acesso à leitura, ainda que devidamente controlada pela igreja. Contudo, com o desenvolvimento da imprensa escrita e também do mercado do livro no século XVIII na Europa, iniciou-se a história do leitor. Um século depois, na França, surgiram as bibliotecas abertas, que tinham em seus acervos livros de cunho educativo (Chartier \& Hébrard, 1995).

Lajolo e Zilberman (1996) abordam que no Brasil, em razão da própria história da colonização, houve uma demora na expansão da leitura; apenas em 1840, no Rio de Janeiro, se teve notícias do início de uma sociedade leitora. Esse fato se deveu, especialmente, porque esta cidade tinha na época alguns recursos como tipografia, livrarias e bibliotecas abertas. Um século mais tarde, em razão do desenvolvimento socioeconômico, houve um crescimento significativo dos leitores no país. Esse impacto pode ser sentido pelo interesse em se pesquisar a leitura em âmbito nacional a partir da década de 1940.
Na década de 1950, conforme aponta Joly (2001), os estudos sobre a leitura com compreensão ganharam espaço no cenário científico nacional em razão das várias correntes teóricas, em especial à psicologia behaviorista e mais tarde à psicologia cognitiva, que trazia considerações sobre os processos cognitivos envolvidos na percepção e compreensão em leitura. A década de 1970, por sua vez, marcou a contribuição das teorias interacionistas.

A década de 1980 foi caracterizada pela presença da abordagem comunicativa, que considerava a capacidade de o leitor em comportar-se linguisticamente nas diversas situações comunicativas (Gerhardt \& Vargas, 2010). As décadas de 1990 e 2000 são marcadas pelo advento da internet e das novas tecnologias da informação, em que o processo de leitura sofre grande influência desses instrumentos de expressão da informação (Oswald \& Rocha, 2013).

Para Gomes e Boruchovitch (2009), pode-se dizer que os parâmetros encontrados nos currículos de língua portuguesa têm usado um sistema de ensino mais aberto. Nesse modelo, o aluno não tem que recorrer ao comportamento mecânico de memorização e aplicação das regras da língua, mas o que é ensinado é a aplicação destas em distintos contextos nos quais se exigem diferentes expressões da linguagem oral e escrita.

Ainda que tenha havido a atualização da forma de ensino de língua portuguesa, e também ponderando-se o fato de que a leitura esteja invariavelmente presente nos veículos de acesso à informação como a internet, é fato que a qualidade da compreensão em leitura exibida por nossos estudantes ainda se mostra insuficiente. Gomes e Boruchovitch (2009), ao analisarem dados do Ministério da Educação referentes às avaliações do ensino formal num período de 1995 a 2005, concluíram que do ensino fundamental ao ensino médio houve um decréscimo no Brasil do desempenho dos alunos no domínio da leitura e seu letramento. Este quadro não se difere do que encontramos atualmente, tendo-se recentemente observado uma piora dos estudantes brasileiros em língua portuguesa no PISA (Programme for International Student Assessment), que ocuparam a 55 posição entre os países avaliados (Brasil, 2012).

Dados recentes trazidos no estudo de Cárnio, Pereira, Alves e Andrade (2011) evidenciam que 875 milhões de pessoas no mundo não têm domínio da habilidade de leitura. Este quadro torna-se mais crítico quando analisamos as considerações de Joly (2001) que revelam que, dentre as pessoas que sabem ler, muitas apresentam dificuldades quanto à realização da leitura, demonstrando problemas na compreensão do conteúdo lido. Isto corrobora com as informações em âmbito nacional dos dados do Indicador de Alfabetismo Funcional, que revelam que somente $28 \%$ da população brasileira pode ser considerada realmente letrada (Cárnio \& cols., 2011).

A situação no contexto nacional em parte se assemeIha ao quadro mundial no que tange ao acesso à educação. No entanto, sob essa perspectiva, Gomes e Boruchovitch (2009) observam que embora tenha havido aumento do percentual de jovens que concluem o ensino fundamental 
no Brasil, aspectos como a evasão e a repetência sofreram uma mudança de nomenclatura, qual seja, escolarização sem aprendizagem (isso implica que o estudante conclui a escolaridade, mas não necessariamente adquire as habilidades e competências que seriam esperadas).

A partir do exposto, nota-se que o desempenho do leitor brasileiro em termos de compreensão e leitura mostra-se crítico e isto se estende para todos os níveis da educação. Por exemplo, Santos, Boruchovitch e Oliveira (2009) apontam para as dificuldades apresentadas pelos alunos nas diferentes etapas do ensino formal em relação à leitura e sua compreensão. A realidade remete ao fato de que é possível observar, muitas vezes, pessoas que ingressam no ensino superior com sérias dificuldades de compreensão leitora, com desempenho muito aquém do esperado para esse nível de escolaridade. Dentre os problemas encontrados, ressaltam-se dificuldades de vocabulário, de compreensão do material lido e uma leitura disfluente (i. e., ausência de automaticidade e de prosódia (Navas, Pinto, \& Dellisa, 2009)).

Ler é uma atividade complexa que exige o uso do processamento cognitivo para que se realize. Requer também uma dinâmica interação entre o leitor e o texto lido e, por meio dessa articulação que abarca uma série de processamentos cognitivos, a leitura se concretiza e vai sendo aprimorada, pois ela funciona com retroalimentação. Por isso, considera-se que ler não é tarefa fácil, haja vista que requer uma rede de processamentos que devem funcionar em perfeita combinação (Santos \& cols., 2009). Face às considerações apresentadas, cabe discutir o processo de compreensão em leitura, pois nele ficam evidenciadas quais são as habilidades e os processamentos que o leitor deve dominar para apresentar uma leitura proficiente. Para tanto, esse é o foco do próximo assunto a ser tratado.

\section{Compreensão em Leitura e seu Processamento}

Existem quatro concepções que fundamentam as pesquisas acerca dos processos de compreensão de leitura. A primeira considera que a habilidade de leitura está associada a uma complexa interação da cognição, da linguística e dos processos perceptuais. A segunda, além das interações entre cognição, linguística e percepção, a emoção também seria um aspecto que se relacionaria à compreensão. A terceira defende que a habilidade de processamento textual da informação é tida como fundamental para que a compreensão ocorra. Por fim, a quarta concepção entende que a compreensão em leitura é adquirida por meio do uso de estratégias direcionadas ao aprendizado (Hall, 1989).

Na visão de Navas e cols. (2009), ler um texto e exibir compreensão é uma tarefa complexa, pois a leitura exige muito mais do que a decodificação das palavras, sendo que o foco essencial é a compreensão do material como um todo. Por isso, a decodificação das palavras pode ser entendida como uma ação necessária, mas por si só não seria suficiente para que a compreensão ocorresse. Assim sendo, para haver compreensão em leitura se faz necessário o uso de habilidades cognitivas que envolvem a realização de inferências do conteúdo lido, o domínio linguístico e semântico e o conhecimento do vocabulário.

Em diversos trabalhos, Kintsch (p. ex., 1988, 1994; Van Dijk \& Kintsch, 1983) destaca o papel do contexto, das estratégias e da atividade do sujeito para a compreensão leitora, em um modelo cognitivo de integração e construção. Segundo este modelo, a representação mental do texto precisa ser construída de forma ativa pelo sujeito, a partir da interpretação das informações que chegam até a ele, não ocorrendo, portanto, de forma automática. Além disso, a construção de uma representação mental significativa exige que o sujeito tenha um conhecimento prévio sobre o assunto e um conjunto de crenças, motivações ou atitudes em relação a ele. A atribuição de significado ao input ocorre de maneira estratégica, ou seja, o sistema cognitivo utiliza diferentes recursos para atingir o objetivo de criar uma representação efetiva do discurso e isto ocorre tão logo a informação é processada.

Sob esse aspecto, Kintsch (1994) aborda que o conhecimento prévio (background knowledge) está diretamente relacionado à compreensão e à aprendizagem. $\mathrm{O}$ arcabouço de conhecimento prévio de uma pessoa poderia explicar porque alguns compreendem melhor um determinado texto, enquanto outras demonstram maior dificuldade. Além disso, a aprendizagem (quando uma pessoa passa a conhecer mais, pode-se induzir que ela aprendeu e que a nova informação adquirida poderá ser empregada em outros contextos) e a memória (processo que antecede a aprendizagem, sendo um pré-requisito para que ela ocorra) são essenciais para que a compreensão se concretize. Nessa linha, McNamara, Sternberg e Hardy (1991) consideram que a compreensão pode ser tida como um comportamento verbal que exige domínio do vocabulário e também uma ampla variedade de conhecimentos prévios que estão diretamente associados ao êxito na realização da Leitura.

Van Dijk e Kintsch (1983) consideram que há níveis de conhecimento no processamento cognitivo da compreensão textual, quais sejam, microestrutura e macroestrutura. O processamento da microestrutura é caracterizado pelos conhecimentos mais específicos e refinados acerca de um determinado assunto, enquanto que o processamento da macroestrutura seria mais global. Desse modo, para a composição da coerência local (conexões significativas entre sentenças sucessivas no discurso), são utilizadas as microestratégias, que atuam na memória de curto prazo por intervenção do sistema de controle. As macroestratégias são utilizadas para inferir o sentido geral do que foi lido (o que forma a macroestrutura textual). A macroestrutura se difere do texto base por conter somente os pontos essenciais do discurso, enquanto que este último apresenta o texto em todos os seus detalhes.

Para Bzuneck (2004), a aprendizagem de uma nova informação envolveria inicialmente a memória sensorial que representaria a percepção seletiva da pessoa frente a uma nova informação. Assim, a pessoa recebe a informação, processa e a encaminha para uma memória de trabalho 
(que armazenaria de forma temporária as informações para que sejam usadas em situações futuras). Na sequência, a informação processada passaria para a memória de longa duração, onde a informação ficaria arquivada, aguardando ser utilizada para a compreensão de novas informações que ocorram em diferentes situações e experiências. Outros dois aspectos relevantes no processamento da informação são apontados nos trabalhos de Atkinson e Shiffrin (1971) e Johson e Hasher (1987) que consideram a atenção (papel de manter a informação ativada) e a lembrança (papel de recuperar e reconstrução da informação) elementos essenciais no processamento, especialmente na realização de uma leitura.

Assim sendo, a compreensão da leitura pode ser concebida como um processo cognitivo que envolve dois processadores, quais sejam, bottom-up e top-down. No primeiro caso, a compreensão se daria por meio da decodificação e do reconhecimento linguístico. No segundo, exige-se contextualização e atribuição de significado à leitura (Nicholson, 1998).

Segundo Nicholson (1998), no processador conhecido como bottom-up, o leitor realiza uma análise do significado individual das palavras do texto, sendo que cada uma é decodificada, para então haver compreensão linguística. Nesse processador, existiria um "Decodificador" (Decoder) cujo papel seria converter a linguagem escrita numa representação fonológica abstrata. A sequência do processo se daria por meio de uma estrutura conhecida como "Bibliotecário" (Librarian) cuja função seria acessar o dicionário mental, isto é, conhecimentos previamente armazenados, para que seja atribuído significado sintático e semântico. A informação é, de fato, armazenada quando é encaminhada para a memória de longo prazo. Quanto ao processador top-down, ele seria responsável por uma compreensão governada pelo entendimento contextual, cuja principal vantagem é a redução da quantidade de informação a ser processada. Desse modo, neste tipo de processamento não haveria necessidade de se conhecer e decodificar cada palavra do texto, pois a leitura é realizada de modo automático e a compreensão obtida por meio de processos de inferências e predição (fazendo-se uso de seu conhecimento prévio, de pistas contextuais e de informações sintáticas e semânticas presentes no texto).

Autores como Gilabert, Martínez e Vidal-Abarca (2005) e Guterman(2003) ressaltam ainda que a compreensão em leitura depende de metacompreensão, que seria a capacidade de monitorar e regular o próprio aprendizado. Dessa forma, leitores proficientes seriam aqueles que conseguem obter o máximo de aproveitamento da leitura realizada. Demonstram um bom funcionamento das estruturas cognitivas e seus processadores, bem como capacidade de compreender quando não entenderam determinada informação a fim de regular o comportamento enquanto leem para que a informação enfim seja entendida.

Com base nos apontamentos trazidos nesta seção, fica claro que investigar a habilidade de compreensão em leitura não é tarefa fácil, pois exige a consideração simul- tânea de múltiplos aspectos do processo cognitivo. Assim, na sequência, serão apresentadas algumas das principais formas utilizadas para se mensurar a compreensão da leitura, sendo que outras habilidades ou comportamentos relacionados não serão enfatizados (como o reconhecimento de palavras e a decodificação). Outro ponto a se mencionar é que, por vezes, a mensuração da habilidade de compreensão leitora não é feita por meio de testes diagnósticos, construídos a partir parâmetros da psicometria, mas, sim, a partir de tarefas/atividades que visam à observação qualitativa dessa habilidade. Desta forma, como opção metodológica, este trabalho se centra apenas no primeiro grupo de instrumentos.

\section{Avaliação da Compreensão em Leitura}

Os testes psicométricos, conhecidos como testes de habilidades, atitudes, interesses, caráter, valores e personalidade, podem ter finalidades diferenciadas, como a mensuração de conhecimentos gerais ou específicos, aptidões psicológicas e práticas (Urbina, 2014). Questionários, inventários e escalas são os tipos mais comuns dos testes psicométricos, trazendo perguntas ou afirmativas acerca de determinado tema. O principal objetivo seria o levantamento de informações ou perfil de uma determinada pessoa dentro de um dado contexto social.

No presente estudo, o contexto psicoeducacional serve de parâmetro para as discussões acerca da compreensão em leitura, haja vista que Santos e cols. (2009) argumentam que a dificuldade de compreensão em leitura é o que está por trás dos encaminhamentos dos estudantes para avaliação. O diagnóstico da compreensão em leitura é muito importante, mas como ler é um comportamento complexo, Salvia e Ysseldyke (1991) observam que não há testes diagnósticos de leitura com valor preditivo suficientemente capaz de mensurar todos os fatores implicados na expressão dessa habilidade. Os testes com finalidade diagnóstica, de uma forma geral, levantam somente uma habilidade relacionada à leitura e sua compreensão.

Nessa direção, Salvia e Ysseldyke (1991) ainda destacam que os testes diagnósticos têm a finalidade de averiguar seis aspectos associados à habilidade de leitura: compreensão literal (identificação de elementos que aparecem de forma evidente no texto); compreensão inferencial (interpretação e aplicação e aproveitamento futuro do conteúdo lido); compreensão auditiva (capacidade de rememoração da informação ouvida para que então a resposta seja dada, sendo que tais testes podem incluir também a compreensão literal e inferencial); compreensão crítica (capacidade de avaliar e emitir opinião sobre o conteúdo lido); compreensão lexical (requer conhecimento de palavras-chave do vocabulário e do significado de palavras específicas do texto) e, por fim, estão os testes de compreensão afetiva (há necessidade de respostas pessoais e emocionais que o sujeito emite quanto ao conteúdo lido). 
No que tange aos testes direcionados ao levantamento da compreensão em leitura, Santos e cols. (2009) consideram que esses instrumentos têm como foco a avaliação do nível de compreensão em leitura e a identificação das dificuldades inerentes ao comportamento de ler. Assim sendo, ao se realizar uma avaliação da compreensão em leitura de um estudante, devem-se utilizar testes que tenham os estudos psicométricos necessários para que possam ser empregados de forma confiável.

Sendo assim, nesta seção são revisados os principais instrumentos de avaliação da compreensão leitora publicados nos contextos internacional e nacional. Nosso foco será a descrição dos objetivos e formatos desses instrumentos, além de considerações acerca das suas características psicométricas.

\section{Instrumentos de Medidas Internacionais}

Criado em 1973, o Woodcock Johnson Reading Mastery Tests está atualmente em sua terceira edição e constitui um dos instrumentos mais utilizados para avaliação das habilidades de leitura na língua inglesa, sendo indicado para indivíduos de dois a 90 anos de idade (Woodcock, Schrank, Mather, \& McGrew, 2007; Woodcock, 1997). O teste é composto por duas formas (não paralelas) contendo subtestes que avaliam várias habilidades subjacentes à leitura (como reconhecimento de letras e de palavras), sendo que a compreensão é avaliada em dois subtestes: compreensão de palavras (vocabulário) e compreensão de passagens. O teste de compreensão de palavras é composto por três seções, sendo que as duas primeiras solicitam que o sujeito dê o sinônimo ou antônimo de palavras lidas, enquanto que a última exige a extração de analogias a partir de pares de palavras. O formato do subteste de compreensão de sentenças é no estilo Cloze (completar o texto lacunado), com a presença de pistas contextuais fornecidas por figuras em dois terços dos itens mais fáceis. Há normas para os Estados Unidos e Canadá e, recentemente, o teste foi traduzido e adaptado para o contexto brasileiro (Wechsler $\&$ cols., 2010). Apesar de sua reconhecida utilidade, o teste tem recebido críticas em relação às suas propriedades psicométricas, principalmente no que diz respeito às questões da validade (Grenwelge, 2009; Rose, 1999).

Outro teste largamente utilizado nos Estados Unidos é o The Gray Oral Reading Tests (GORT; Wiederholt \& Bryant, 2012), agora em sua quinta edição. Os principais usos informados no manual constituem a identificação de dificuldades de leitura, o diagnóstico de dificuldades específicas de aprendizagem, a identificação de forças e fraquezas do avaliando e investigação dos progressos na leitura. O teste apresenta duas formas paralelas (A e B), contendo cada uma delas 14 passagens apresentadas em ordem crescente de dificuldade, seguidas por cinco questões de compreensão no formato de múltipla escolha. As normas do teste incluem a faixa etária dos seis aos 23 anos e 11 meses e há indicadores psicométricos de validade e fidedignidade.
Entretanto, um estudo demonstrou problemas quanto à validade do instrumento. Keenan e Betjemann(2006) solicitaram a um grupo de crianças que respondessem ao teste sem lerem as passagens. Como resultados, os autores descobriram que as crianças endossaram as respostas corretas de maneira significativamente maior do que seria esperado pelo acaso. Ademais, análises comparando os itens dependentes da leitura das passagens versus os que independiam das passagens mostraram que os últimos não são sensíveis às dificuldades de aprendizagem, além de não correlacionarem com o desempenho em outros testes de compreensão. A partir dos resultados, as autoras questionam as validades concorrente e de conteúdo do GORT, recomendando ponderações em seu uso.

O Peabody Individual Achievement Test (PIAT) é um teste de desempenho escolar normatizado para alunos dos cinco aos 18 anos de idade. A edição atual é uma revisão (PIAT-R) da versão original criada em 1970 (Markwardt, 1998). Composto por seis subtestes, a compreensão da leitura é avaliada pela capacidade do estudante em extrair significados de sentenças lidas silenciosamente, escolhendo uma de quatro figuras que mais bem as representa. Apesar de terem sido relatados problemas com o subteste de escrita em termos de validade e fidedignidade (Luther, 1992), os demais subtestes têm apresentado, consistentemente, indicadores psicométricos adequados (p. ex., Franzen, 2000; Soethe, 1972).

Talvez menos conhecido no contexto brasileiro, o Wechsler Individual Achievement Test-Third Edition (WIAT-III; Wechsler, 2009) é um instrumento clínico de realização disponível para indivíduos entre quatro e 50 anos de idade. É composto por subtestes que avaliam várias habilidades, tais como a expressão escrita, a aritmética e a compreensão oral. O subteste de compreensão leitora constitui uma medida das habilidades de compreensão literal e inferencial. O teste apresenta bons indicadores de fidedignidade (teste-reteste, consistência interna e concordância entre avaliadores) e validade (Ackerman, 1998).

Por fim, um dos testes mais tradicionais e utilizados no contexto educacional dos Estados Unidos (particularmente na seleção de alunos nas Universidades) é o Nelson Denny Reading Test (NDRT), padronizado para avaliar as habilidades de vocabulário e compreensão de texto de estudantes do Ensino Médio e Superior (Brown, Fishco, \& Hanna, 1993). Criado em 1929, o formato dos itens permanece praticamente o mesmo, apesar das adaptações posteriores. O subteste de compreensão apresenta um conjunto de passagens (de cinco a oito) com questões de múltipla escolha relacionadas, que devem ser respondidas no tempo limite de 20 minutos. Apresenta como vantagens a rápida e fácil aplicação (que pode ser individual ou coletiva) e a existência de formas paralelas. Apesar de o teste possuir bons indicadores psicométricos, tem-se apontado problemas relacionados à validade (p. ex., Coleman, Lindstrom, Nelson, Lindstrom, \& Gregg, 2010) e precisão (Murray-Ward, 1998).

Em síntese, verifica-se que os principais testes estrangeiros utilizados no contexto do diagnóstico da compre- 
ensão leitora apresentam como vantagens a utilização de amostras normativas amplas e a presença de estudos psicométricos atestando para suas características de validade e fidedignidade. Entretanto, estudos independentes relatados na literatura nem sempre confirmam as propriedades relatadas nos manuais e, muitas vezes, pelo contrário, as questionam. Por exemplo, a correlação entre alguns testes listados nesta sessão varia de baixa a moderada, podendo-se questionar se avaliam o mesmo construto (Keenan, Betjemann, \& Olson, 2008; Ott, 2010). Por fim, como desvantagens desses instrumentos, destaca-se a ausência de variabilidade no formato dos itens de teste, que frequentemente apresenta-se na forma de questões de múltipla escolha ou de Cloze, o que descaracteriza a natureza multidimensional da compreensão leitora (Israel \& Duffy, 2008). Assim, concentrar a avaliação em apenas algumas habilidades envolvidas no ato de compreender enfraquece o diagnóstico, pois prejudica os que apresentam falhas apenas nas habilidades investigadas e oculta as dificuldades daqueles que apresentam problemas somente em outras.

\section{Instrumentos de Medidas Brasileiros}

A realidade da avaliação da compreensão leitora no Brasil em muito se distancia do que foi apresentado para os instrumentos internacionais. O quadro atual remete à praticamente inexistência de instrumentos formais de avaliação da compreensão, que apresentem indicadores de qualidade psicométrica e sejam disponibilizados por editoras. Estudos recentes apontam para a criação de uma bateria de avaliação da compreensão leitora para crianças brasileiras, com indicadores psicométricos de validade, precisão e normas (Lúcio, Kida, Carvalho, Cogo-Moreira, \& Ávila, 2015; no prelo). Apesar da relevância da iniciativa, há longo caminho a percorrer até que estes instrumentos estejam disponíveis para uso. Assim, a quase totalidade dos procedimentos de avaliação encontrados está dispersa em teses, dissertações ou artigos científicos (Gomes \& Boruchovitch, 2005; Spinillo \& Mahon, 2007), que acabam sendo a única fonte de recursos dos profissionais que trabalham com avaliação.

Mesmo quando os instrumentos são comercializados, não deixam de apresentar problemas. Por exemplo, Saraiva, Moojen e Munarski (2006) propõem a avaliação da compreensão de textos expositivos durante e após a leitura oral e silenciosa, mas não há pesquisas empíricas atestando para as suas características psicométricas, estatísticas descritivas ou normas. Por outro lado, há casos em que ocorre o inverso, ou seja, o instrumento possui indicadores psicométricos, mas não está disponível para uso. Por exemplo, o teste de Cloze apresenta vários estudos de validação no contexto brasileiro (Cunha \& Santos, 2009; Santos \& cols., 2009; Santos, Sisto, \& Noronha, 2010), mas nenhuma de suas formas é comercializada ainda, estando disponível apenas em artigos científicos e capítulos de livros.

Dois subtestes da Bateria PROLEC (Compreensão de Orações e Compreensão de Textos) constituem atu- almente os únicos recursos formais que dispomos para a avaliação da compreensão leitora, apresentando normas intragrupo e estudos de fidedignidade e validade (Cuetos, Rodrigues, \& Ruano, 2012). Apesar do avanço, uma análise mais detalhada do manual mostra a possibilidade de efeito de teto nos subtestes, o que pode prejudicar a variabilidade dos escores e, consequentemente, o diagnóstico de indivíduos com dificuldades de compreensão. Desse modo, o instrumento carece de uma análise cuidadosa dos itens. Além disso, para a fidedignidade do conjunto de provas, foi reportado apenas o alfa de Cronbach. Finalmente, em relação à validade, Oliveira e Capellini (2010) conduziram um estudo no Brasil considerando apenas o contraste entre grupos de sujeitos em função do tipo de escola (municipal ou particular), não havendo informações sobre o funcionamento dos itens de acordo com a escolaridade ou da idade, o que seria esperado em tarefas do desenvolvimento.

Pelo exposto, pode-se observar uma lacuna na pesquisa brasileira na área de construção de testes de compreensão de textos, cujo reflexo é a quase inexistência de instrumentos que forneçam os parâmetros normais de sua aquisição, além de critérios diagnósticos para o desenvolvimento inadequado desta habilidade. Simultaneamente, os instrumentos que hoje são comercializados (Cuetos \& cols., 2012; Saraiva \& cols., 2006) podem não apresentar dados empíricos que deem subsídio às interpretações pretendidas pelos resultados dos escores nas tarefas. Esse quadro, aliado à repercussão que o tema apresenta tanto para fins educacionais quanto para a intervenção clínica, aponta para a necessidade urgente de iniciativas que busquem criar instrumentos de avaliação da compreensão, baseados em evidências.

\section{Considerações Finais}

A avaliação da compreensão em leitura é um tema central para a psicologia educacional, pois as dificuldades inerentes à realização da leitura com compreensão constituem um problema mundial (Cárnio \& cols., 2011; Joly, 2001). Assim, como a leitura ainda é uma das principais causas do fracasso escolar (Gomes \& Boruchovitch, 2009), torna-se relevante discutir a leitura com compreensão, bem como formas de se efetuar a sua avaliação.

Estes foram justamente os objetivos que nortearam a construção do presente trabalho. Por meio de uma revisão da literatura, buscou-se traçar uma exposição geral sobre o estudo da compreensão leitora, desde seus aspectos históricos (tendo como foco a realidade brasileira), até os aspectos conceituais e práticos, estes últimos representados por uma síntese dos principais instrumentos atualmente publicados para a avaliação da compreensão na leitura. De um modo geral, foi possível concluir que a compreensão leitora é um assunto complexo, que envolve a contribuição teórica de profissionais de diversas áreas e, consequentemente, apresenta diferentes perspectivas e pontos de vista teóricos. Adotou-se, no presente estudo, a visão de que a compreen- 
são da leitura envolve os aspectos cognitivos, linguísticos, textuais e socioculturais. Por fim, demonstrou-se que o diagnóstico das dificuldades de compreensão é dificultado não somente pela complexidade do assunto, como pela carência de instrumentos que têm como base de sua construção os parâmetros da psicometria. Nessas considerações finais, pretende-se discutir a repercussão dessas questões para a avaliação no contexto psicoeducacional.

Um fator importante a ser mencionado é que, quando a dificuldade de compreensão em leitura é diagnosticada precocemente, programas interventivos podem ser traçados para minimizar ou reverter a situação. Entretanto, a falta de testes validados e fidedignos dificulta este diagnóstico precoce (Santos \& cols., 2009). Na mesma linha, o desconhecimento por parte de profissionais sobre a necessidade de se empregar meios que apresentem estudos psicométricos que evidenciem a eficácia do recurso diagnóstico empregado na avaliação da compreensão em leitura pode ser um problema, como destacado por Santos e cols. (2009). O resultado de uma avaliação com um recurso que não apresenta evidências de sua validade diagnóstica pode não refletir a real dificuldade apresentada pelo avaliando. Esse fato parece óbvio, mas cabe sua discussão, pois ao se realizar o levantamento as formas de se mensurar a compreensão em leitura neste trabalho, poucos recursos foram encontrados. Paralelamente, dos recursos que foram observados, invariavelmente alguns deles não apresentavam respaldo científico.

A necessidade de se avaliar a compreensão em leitura assume importância para aqueles que se comprometem com as dificuldades inerentes ao processo de aprendizagem, no ensino da leitura e compreensão de textos, sobretudo quando se trata de fazer com que o aluno se torne um bom leitor. Nesse sentido, o maior número de contribuições sobre a mensuração da compreensão em leitura está nas áreas de psicologia, linguística, educação e fonoaudiologia. Também se constatou a presença de muitos estudos sobre da avaliação da compreensão em leitura, mas que fugiram aos nossos propósitos (lembrando que, a este respeito, o objetivo do presente trabalho foi apresentar algumas das principais formas de se mensurar a compreensão em leitura e não trazer estudos que focaram a compreensão em leitura e fatores como sexo, idade, ano escolar ou relações com outras variáveis psicoeducacionais).

Ter uma boa compreensão em leitura é de suma relevância no contexto da aprendizagem. No início da escolarização, a compreensão em leitura assegura um desempenho escolar satisfatório e, se essa habilidade se aprimorar ao longo da escolarização, provavelmente, o aluno irá conseguir percorrer a educação formal sem grandes dificuldades. Destarte, por se tratar de tão importante habilidade, deveria haver mais estudos que pudessem construir formas eficazes de se avaliar a compreensão em leitura, possuindo estas evidências de sua validade e precisão. Posto isto, chama atenção a urgente necessidade de que testes/recursos sejam desenvolvidos em contexto nacional, levando-se em consideração as especificidades das diferentes regiões brasileiras.

\section{Referências}

Ackerman, T. A. (1998). Review of the Wechsler Individual Achievement Test. Em J. C. Impara \& B. S. Plake (Eds.), The thirteenth mental measurement yearbook (pp. 1125-1128). Lincoln, NE: University of Nebraska Press.

Atkinson, R.C., \& Shiffrin, R.M. (1971). The control of short-term memory. Scientific American, 255(2), 82-90.

Brasil. (2012). Relatório Nacional PISA 2012: resultados brasileiros (p. 66). São Paulo: Fundação Santilhana.

Brown, J. I., Fishco, V.V., \& Hanna, G.S. (1993). The Nelson-Denny reading test. Itasca, IL: The Riverside Publishing Company.

Bzuneck, J.A. (2004). Aprendizagem por processamento da informação: uma visão construtivista. Em E. Boruchovitch \& J. A. Bzuneck (Orgs.), Aprendizagem: processos psicológicos e contextos social na escola (pp. 17-54). Petrópolis: Vozes.

Cárnio, M.S., Pereira, M.B., Alves, D.C., \& Andrade, R.V. (2011). Letramento escolar de estudantes de $1^{\text {a }}$ e $2^{\text {a }}$ séries do ensino fundamental de escola pública. Revista da Sociedade Brasileira de Fonoaudiologia, 16(1), 1-8.

Chartier, A.M., \& Hébrard, J. (1995). Discursos sobre a leitura - 18801980. (O. Biato \& S. Bath, Trads.) São Paulo: Ática.

Coleman, C., Lindstrom, J., Nelson, J., Lindstrom, W., \& Gregg, N. (2010). Passageless comprehension on the Nelson Denny Reading Test: Well above chance for university students. Journal of Learning Disabilities, 43(3), 244-249.

Cuetos, F., Rodrigues, B., \& Ruano, E. (2012). Manual das provas de avaliação dos processos de leitura - PROLEC (S.A. Capellini, A. M. de Oliveiria \& F. Cuetos, Trad. e Adapt.) ( $2^{a}$ ed.). São Paulo: Casa do Psicólogo.

Cunha, N. de B. \& Santos, A.A.A. dos. (2009). Validade por processo de resposta no teste de Cloze. Fractal: Revista de Psicologia, 21(3), 549-562.

Franzen, M.D. (2000). Reliability and validity in neuropsychological assessment (2nd edition). New York: Kluwer Academic/Plenum Publishers.

Gerhardt, A.F.L.M., \& Vargas, D.S. (2010). A pesquisa em cognição e as atividades escolares em leitura. Trabalhos em Línguística Aplicada, 49(1), 145-166.

Gilabert, R., Martínez, G., \& Vidal-Abarca, E. (2005). Some good text are always better text revision to foster inferences of readers 
with high and low prior background knowledge. Learning and Instruction, 15(1), 45-68.

Gomes, M.A.M., \& Boruchovitch, E. (2005). Desempenho no jogo, estratégias de aprendizagem e compreensão na leitura. Psicologia: Teoria e Pesquisa, 21(3), 319-326.

Gomes, M.A.M., \& Boruchovitch, E. (2009). Proficiência em leitura: um panorama da situação. Em A. A. A. dos Santos, E. Boruchovitch, \& K. L. de Oliveira (Orgs.), Cloze: um instrumento de diagnóstico e intervenção (pp. 23-46). São Paulo: Casa do Psicólogo.

Grenwelge, C.H. (2009). Test review: Woodcock, R. W., Schrank, F. A., Mather, N., \& McGrew, K. S. (2007). "Woodcock-Johnson III Tests of Achievement, Form C/Brief Battery." Rolling Meadows, IL: Riverside. Journal of Psychoeducational Assessment, 27(4), 345-350.

Guterman, E. (2003). Integrating written metacognitive awareness guidance as a "psychological tool" to improve student performance. Learning and Instruction, 13(6), 633-651.

Hall, W.S. (1989). Reading comprehension. American Psycologist, 44(2), 157-161.

Israel, S.E., \& Duffy, G.G. (Orgs.). (2008). Handbook of research on reading comprehension. New York: Routledge.

Johson, M.K. \& Hasher, L. (1987). Human learning and memory. Annual Review of Psychology, 38, 361-368.

Joly, M.C.R.A. (2001). Leitura no contexto educacional: avaliando estratégias para aquisição de habilidades criativas. Em F. F. Sisto, E. T. B. Sbardelini, \& R. Primi (Orgs.), Contextos e questões da avaliação psicológica (pp. 99-116). São Paulo: Casa do Psicólogo.

Keenan, J.M. \& Betjemann, R.S. (2006). Comprehending the gray oral reading test without reading it: Why comprehension tests should not include passage-independent items. Scientific Studies of Reading, 10(4), 363-380.

Keenan, J.M., Betjemann, R.S., \& Olson, R.K. (2008). Reading comprehension tests vary in the skills they assess: Differential dependence on decoding and oral comprehension. Scientific Studies of Reading, 12(3), 281-300.

Kintsch, W. (1988). The role of knowledge in discourse comprehension: A construction-integration model. Psychological Review, 95(2), 163-82.

Kintsch, W. (1994). Text comprehension, memory, and learning. American Psycologist, 49(4), 294-303.

Lajolo, M. \& Zilberman, R. (1996). A Formação da Leitura no Brasil. São Paulo: Ática.

Lucio, P.S., Kida, A.S.B., Carvalho, C.A.F., Cogo-Moreira, H., \& Ávila,
C.R.B. (no prelo). Estudo de Fidedignidade do Avaliador em Provas de Compreensão Leitora e Oral. Revista Avaliação Psicológica.

Lucio, P.S., Kida, A.S.B., Carvalho, C.A.F., Cogo-Moreira, H., \& Ávila, C.R.B. (2015). Construção de uma prova para avaliação da compreensão leitora no ensino fundamental: Estudo piloto. Temas em Psicologia, 23(4), 1035-1050.

Luther, J.B. (1992). Review of the Peabody Individual Achievement Test-Revised. Journal of School Psychology, 30(1), 31-39.

Markwardt, F.C. (1998). Peabody individual achievement test (Rev. ed.). Circle Pines, MN: AGS Publishing.

McNamara, T.P., Sternberg, R.J., \& Hardy, J.K. (1991). Processing verbal relations. Intelligence, 13, 193-221.

Murray-Ward, M. (1998). Review of the Nelson-Denny Reading Test, Forms G and H. Em J. C. Impara \& B. S. Plake (Eds.), The thirteenth mental measurement yearbook (pp. 683-685).

Navas, A.L.G.P., Pinto, J.C.B.R., \& Dellisa, P.R.R. (2009). Avanços no conhecimento do processamento da fluência em leitura: da palavra ao texto. Revista Da Sociedade Brasileira de Fonoaudiologia, 14(4), 553-559.

Nicholson, T. (1998). Reading comprehension processes. Em G. B. Thompson \& T. Nicholson (Eds.), Learning to read: Beyond phonics and whole language (pp. 127-149). New York: Teachers College Press.

Oliveira, A.M. De, \& Capellini, S.A. (2010). Desempenho de escolares na adaptação brasileira da avaliação dos processos de leitura. Pró-Fono Revista de Atualização Científica, 22(4), 555-560.

Oswald, M.L. \& Rocha, S.L.A. da. (2013). Sobre juventude e leitura na "idade mídia": implicações para políticas e práticas curriculares. Educar Em Revista, 47, 267-283.

Ott, L.M. (2010). Comparison of the reading subtests of the Wechsler Individual Achievement Test - Third Edition and the Peabody Individual Achievement Test-Revised/Normative Update. (Thesis). Marshall University, Huntington, US. Recuperado: maio de 2014. Disponível: http://mds.marshall.edu/cgi/viewcontent. cgi?article=1115\&context=etd.

Rose, M. De. (1999). A Review of the Woodcock Reading Mastery. TESL Canada Journal/Review TESL Du Canada, 16(2), 86-93.

Salvia, J., \& Ysseldyke, J. E. (1991). Avaliação em educação especial e corretiva ( $4^{\mathrm{a}}$ ed.). São Paulo: Manole.

Santos, A.A.A., Boruchovitch, E., \& Oliveira, K. L. (2009). Cloze: um instrumento de diagnóstico e intervenção. São Paulo: Casa do Psicólogo.

Santos, A.A.A. dos, Sisto, F.F., \& Noronha, A.P.P. (2010). TONI 3 - 
Forma A e Teste de Cloze: evidências de validade. Psicologia: Teoria E Pesquisa, 26(3), 399-405.

Saraiva, R.A., Moojen, S.M.P., \& Munarski, R. (2006). Avaliação da compreensão leitora de textos expositivos para fonoaudiólogos e psicopedagogos ( $2^{a}$ ed.). São Paulo: Casa do Psicólogo.

Soethe, J.W. (1972). Concurrent validity of the Peabody Individual Achievement Test. Journal of Learning Disabilities, 5(9), 631-634.

Spinillo, A.G. \& Mahon, E.R. (2007). Compreensão de texto em crianças: comparações entre diferentes classes de inferência a partir de uma metodologia on-line. Psicologia: Reflexão E Crítica, 20(3), 463-471.

Urbina, S. (2014). Essentials of psychological testing. New Jersey: John Wiley \& Sons, Inc.

Van Dijk, T.A. \& Kintsch, W. (1983). Strategies of Discourse Comprehension. New York: Academic Press.
Wechsler, D. (2009). Wechsler Individual Achievement Test -Third Edition. San Antonio, TX: NCS Pearson.

Wechsler, S.M., Nunes, C.S., Pasian, S.R., Homsi, S.V., Moretti, L., \& Anache, A.A. (2010). Brazilian adaptation of the WoodcockJohnson III cognitive tests. School Psychology International, 31(4), $409-421$.

Wiederholt, J.L., \& Bryant, B. (2012). Gray oral reading test (5th ed.). Austin, TX: Pro-Ed.

Woodcock, R.W. (1997). Woodcock Reading Mastery Tests-revised/ normative update. Circle Pines, MN: American Guidance Service.

Woodcock, R.W., Schrank, F.A., Mather, N., \& McGrew, K.S. (2007). Woodcock-Johnson III Normative Update Technical Manual. Rolling Meadows, IL: Riverside.

\section{Sobre os autores}

Katya Luciane de Oliveira (katyauel@gmail.com)

Professora Adjunta do Departamento de Psicologia e Psicanálise/PPSIC e do Mestrado em Educação da Universidade Estadual de Londrina.

Patrícia Silva Lúcio (pslucio@gmail.com)

Professora Assistente do Departamento de Psicologia e Psicanálise da Universidade Estadual de Londrina.

Fabiano Koich Miguel (fabiano@avalpsi.com.br)

Professor Adjunto do Departamento de Psicologia e Psicanálise da Universidade Estadual de Londrina. 
